\title{
Conservation et production de la valeur du Fiore
} Sardo

Producteurs, commerçants et activistes dans la régulation de la typicité d'un fromage italien

\section{Valeria Siniscalchi et Franco Zecchin}

\section{CpenEdition}

\section{Journals}

Édition électronique

URL : https://journals.openedition.org/tc/8807

DOI : $10.4000 /$ tc. 8807

ISSN : 1952-420X

Éditeur

Éditions de l'EHESS

\section{Édition imprimée}

Date de publication : 17 mai 2018

Pagination : 56-73

ISBN : 978-2-7132-2750-9

ISSN : 0248-6016

Référence électronique

Valeria Siniscalchi et Franco Zecchin, «Conservation et production de la valeur du Fiore Sardo »,

Techniques \& Culture [En ligne], 69 | 2018, mis en ligne le 17 mai 2020, consulté le 29 septembre 2022.

URL : http://journals.openedition.org/tc/8807 ; DOI : https://doi.org/10.4000/tc.8807 


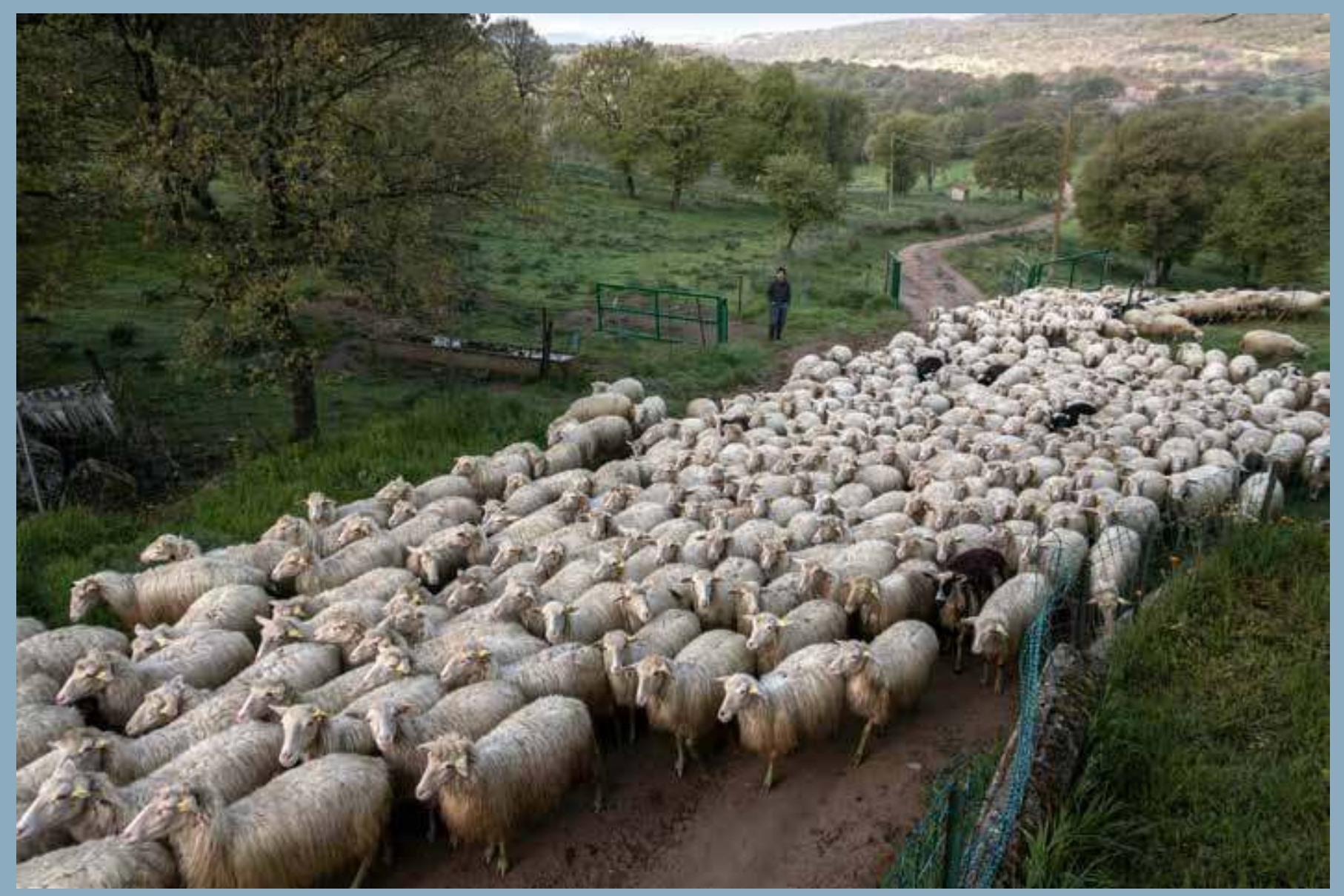




\section{Conservation et production de la valeur du Fiore Sardo}

\section{Producteurs, commerçants et activistes dans la régulation de la typicité d'un fromage italien}

En quoi consiste la conservation d'un fromage? Quelles transformations fait-elle subir au produit? Quels sont ses enjeux? J'aborderai ici les questions liées aux pratiques de conservation et aux processus de valorisation des fromages à travers le cas du Fiore Sardo, en prêtant attention aux rapports de pouvoir entre les différents acteurs impliqués dans ces processus. En effet, ce fromage produit en Sardaigne fait l'objet de différents labels et focalise l'attention de nombreux acteurs: les bergers producteurs, les producteurs industriels, des représentants des institutions régionales, des commerçants locaux tout comme des entrepreneurs internationaux, et enfin les membres du mouvement Slow Food ${ }^{1}$. La définition du véritable Fiore Sardo et la régulation de la production de ce fromage cristallisent des tensions et des conflits aigus que j'analyserai dans ces pages ${ }^{2}$.

La question de la définition de l'authenticité n'est pas nouvelle en anthropologie et a été abordée dans la littérature consacrée aux productions typiques et aux appellations d'origine. Il s'agit d'une littérature extrêmement vaste sur laquelle j'ai déjà eu l'occasion de revenir dans des écrits antérieurs (Siniscalchi 2000, 2010), en soulignant ses apports et ses limites ${ }^{3}$. Une partie des approches plus récentes se rattache, tout en les revisitant, aux analyses d'Appadurai (1986) et de Kopitoff (1986), qui avaient déconstruit la notion de marchandise et proposé de nouvelles clés de lecture des processus de «commodification», c'est-à-dire des processus de transformation de produits en marchandises destinées à l'échange, à travers l'idée que les objets ont une vie sociale dont il est possible de reconstruire la biographie culturelle. D'autres travaux rénovent les approches anthropologiques de la political economy, en prêtant attention aux circulations - des valeurs, des pratiques et des produits - et aux dynamiques politiques qui les caractérisent (Wilk 2006, Meneley 2007, Paxson 2013, Weiss 2016 entre autres). Dans les pages qui suivent, je m'intéresserai plus particulièrement, à partir de ces questions, à la vie du Fiore Sardo ${ }^{4}$ en prêtant attention aux transformations que le fromage subit pendant son affinage et aux enjeux de sa régulation et de sa mise en circulation; j'explorerai également les dynamiques sociales, politiques et économiques qui s'agrègent autour de sa conservation. 


\section{Le champ conflictuel de la régulation du Fiore Sardo}

Originaire de la partie interne de l'île, au cœur de la Barbagia, le Fiore Sardo est l'un des trois pecorini (fromages de brebis) sardes qui bénéficient, depuis 1996, d'une Appellation d'Origine Contrôlée (AOC). Avant l'introduction du règlement européen de 1996, le Fiore avait déjà bénéficié d'une appellation d'origine ${ }^{5}$. L'AOC s'étend à toute la Sardaigne et regroupe environ une vingtaine de bergers producteurs, qui font du Fiore Sardo à partir du lait de leur propre troupeau et le commercialisent sous leur propre nom, et une vingtaine de producteurs industriels. Certains industriels produisent du fromage à partir du lait acheté à différents éleveurs de brebis de race «sarde», d'autres achètent du fromage produit par des bergers producteurs, souvent avant que le label AOC n'y soit apposé (donc avant que la période de cent cinq jours d'affinage ne soit terminée), afin de pouvoir marquer les meules du nom de leur propre entreprise, et parfois après cette période. Chacun des producteurs bergers produit entre 150 et 3000 meules de Fiore Sardo par an $\left(500 \mathrm{~kg}\right.$ à 9 tonnes), en fonction de la taille de son troupeau ${ }^{6}$. En revanche, la production industrielle peut aller, par entreprise, de 18000 meules (60 tonnes) à 70000 meules (270 tonnes) par an.

Une fois obtenue, l'AOC s'est avérée être un champ extrêmement conflictuel où, aujourd'hui encore, ces acteurs s'affrontent par toutes sortes de moyens. Jusqu'en 2012, ces différents groupes de producteurs étaient réunis dans le «Consorzio di tutela» (Groupement de défense), organe prévu par le règlement de l'appellation et chargé de la défense de celle-ci. Mais leurs relations étaient - et sont restées - très tendues. Ces tensions, d'ordre à la fois symbolique et économique, sont liées à la place des uns et des autres dans le marché du Fiore Sardo et à la régulation de ce fromage. L'enjeu pour les producteurs industriels est de pouvoir bénéficier de la renommée de ce fromage considéré comme le plus traditionnel de l'île. Pour les producteurs bergers, en revanche, l'enjeu est la définition du véritable Fiore Sardo et, par-là, la possibilité de différencier et de distinguer leur fromage de celui produit par les industriels. À cause de ces tensions et de ces divergences, les relations que les bergers producteurs entretiennent avec l'AOC sont ambivalentes:

«En 1987, nous n'étions pas encore en AOC. Les producteurs se sont réunis pour rédiger un cahier des charges, mais après, ce sont des institutions [régionales], qui avaient bien d'autres projets en tête, qui ont été déléguées pour le rédiger. Maintenant les industriels produisent du Fiore Sardo, et le consommateur mange le Fiore Sardo des industriels sans qu'il y ait la moindre différence du point de vue réglementaire: leur fromage est protégé au même titre que le nôtre. Nous sommes dans une situation de concurrence à l'américaine. Nous, c'est du lait cru, parce que c'est du lait qui provient d'un seul et même troupeau et il n'y a pas de problème de gonflements » (Antonio A., producteur berger, 27/04/2011).

Les imprécisions du cahier des charges de l'AOC focalisent les critiques des producteurs bergers, qui considèrent l'Appellation d'Origine comme un instrument juridique qui, bien qu'il les défende en empêchant que ce fromage ne soit produit dans d'autres régions, ne les protège 


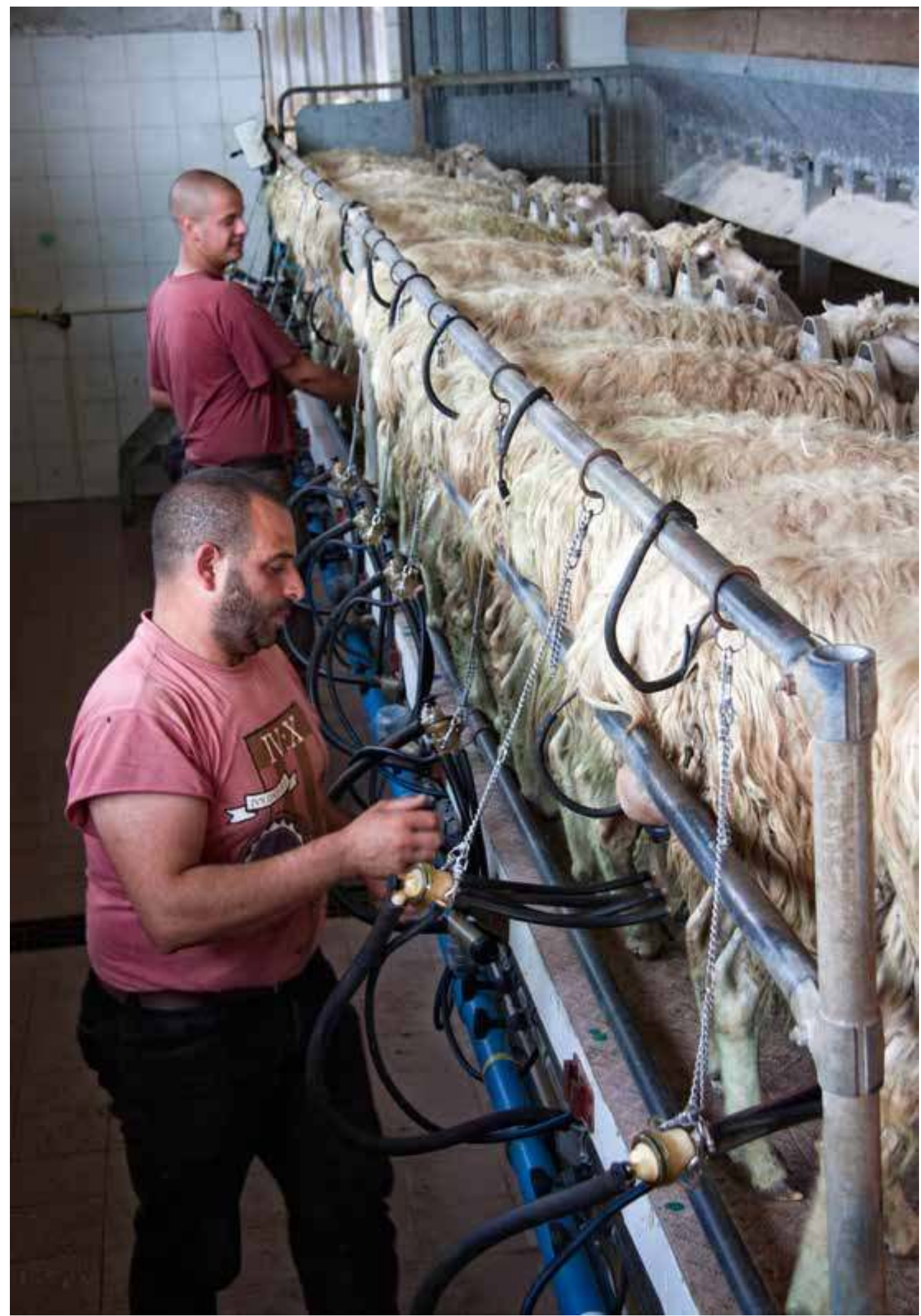

2. La traite des brebis. Selon le cahier des charges de l'AOC, le lait doit être entier, cru, frais et issu de brebis de race sarde. Les différences de charges bactériennes, quand le lait provient de plusieurs troupeaux, entraînent des réactions dans les fromages, ce qui selon les producteurs bergers et certains techniciens, rendrait nécessaire la pasteurisation pour la production industrielle. 


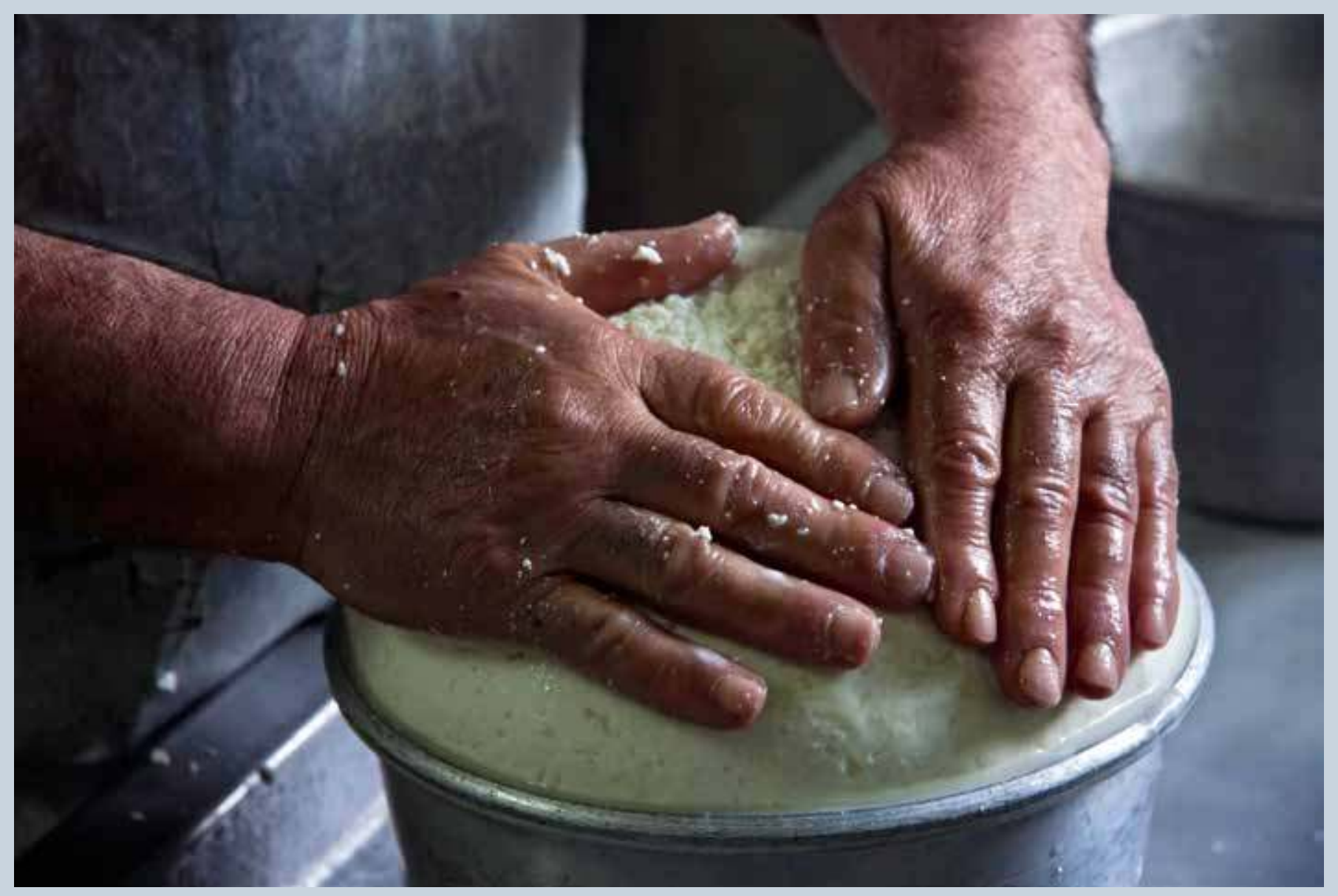

3. Après la rupture du caillé, la pâte de fromage est pressée dans les meules en forme de cône tronqué. 
pas de leur ennemi interne, c'est-à-dire des industriels, qui produisent du Fiore Sardo en bénéficiant tout comme eux du label AOC délivré par le ministère. Certains industriels souhaiteraient également apporter des modifications au texte qui ne sont pas les mêmes que celles voulues par ceux qui produisent le fromage artisanalement. La nature politique de ce cahier des charges apparaît ainsi clairement. Il s'agit d'un texte non consensuel, qui est la résultante d'intérêts très divergents: ceux des producteurs industriels, favorables à un texte suffisamment souple pour ne pas contraindre leur production à grande échelle; ceux des petits producteurs, les bergers producteurs qui, à l'époque de sa rédaction, avaient intérêt à ce que la délimitation de l'aire de production soit à l'échelle de la Sardaigne entière, car tous n'ont pas des terres en Barbagia (même si leurs caves d'affinage se situent généralement dans cette partie de l'île), et qui ont pu obtenir le label AOC grâce au fait que la demande émanait d'un nombre important de producteurs; enfin, les intérêts des institutions régionales, attachées à promouvoir l'ensemble de la production sarde, et pas seulement celle des petits producteurs.

Pour les bergers producteurs, le cahier des charges de l'AOC est trop vague sur des points qu'ils considèrent essentiels pour «distinguer» leur fromage, et donc pour le protéger:

«Les industriels ne fument pas [le fromage]. Ils l'utilisent [le Fiore Sardo] comme un label commercial. [Pour eux et pour le cahier des charges] le fumage est facultatif [...]. On n'est pas en train d'écrire une recette de cuisine, on est en train d'écrire le cahier des charges d'une AOC. Tu dois écrire la réalité telle qu'elle est, point final [...]. Ils devaient écrire que le lait doit provenir d'un seul et même troupeau et que le fumage est obligatoire [...]. Les mêmes [représentants des institutions] qui faisaient l'AOC avec les bergers apprenaient aux industriels comment pasteuriser le lait » (Antonio A., producteur berger, 27/04/2011).

En effet, la possibilité de reconnaître les différences entre le fromage que les producteurs artisanaux considèrent comme le véritable Fiore Sardo et celui qui est produit dans les fromageries industrielles, est un premier niveau de la lutte que ces producteurs mènent dans les bureaux de la Région Sardaigne, à l'intérieur ou à l'extérieur du «Consorzio di tutela», dans les tribunaux (Siniscalchi 2013b), dans les cabinets des experts et dans les bureaux d'études. La définition des techniques de production - parmi lesquelles l'usage du
4. Le surplus de pâte est coupé pour obtenir la forme caractéristique du Fiore Sardo à double tronc de cône.

Le nom de ce fromage est probablement lié aux chardons - et à leurs fleurs - utilisés pour cailler le lait ou, selon d'autres théories, aux fleurs gravées sur le bois des formes portant les initiales du producteur (Mckeown 2016: 272273).

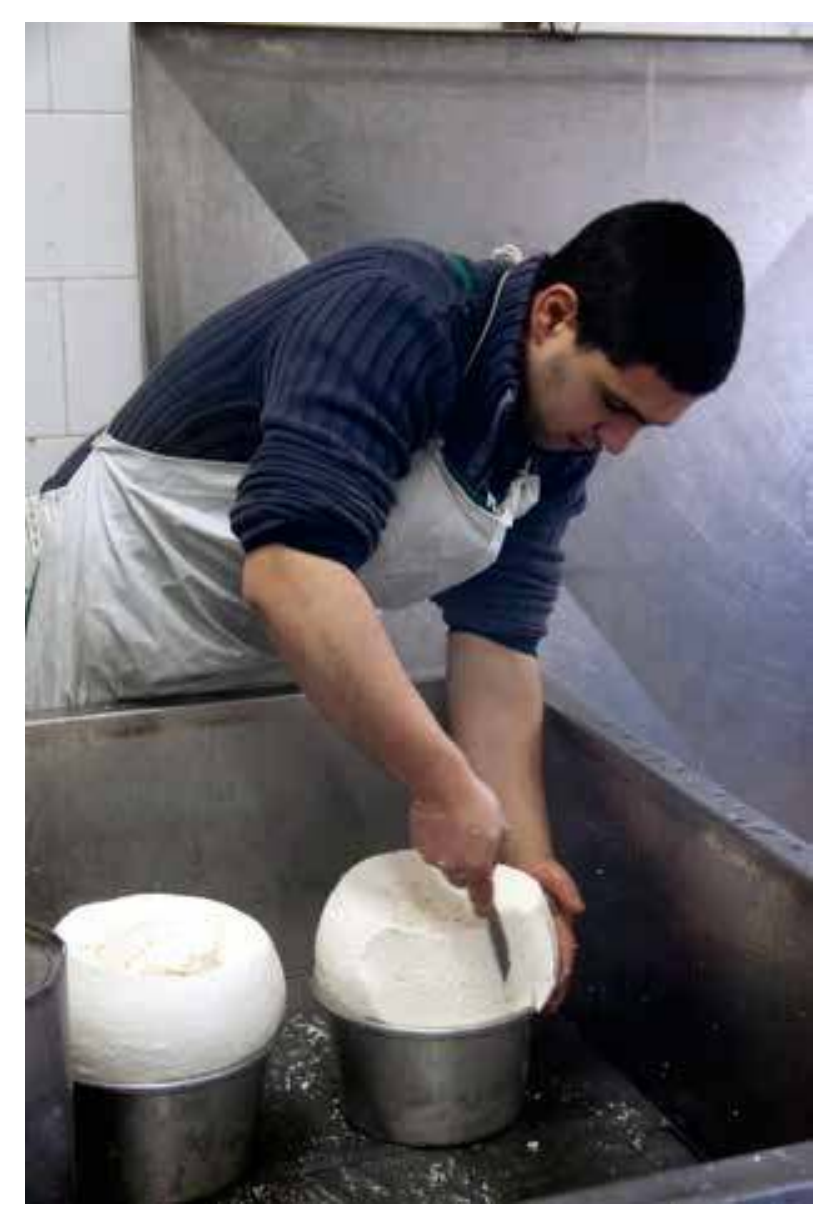




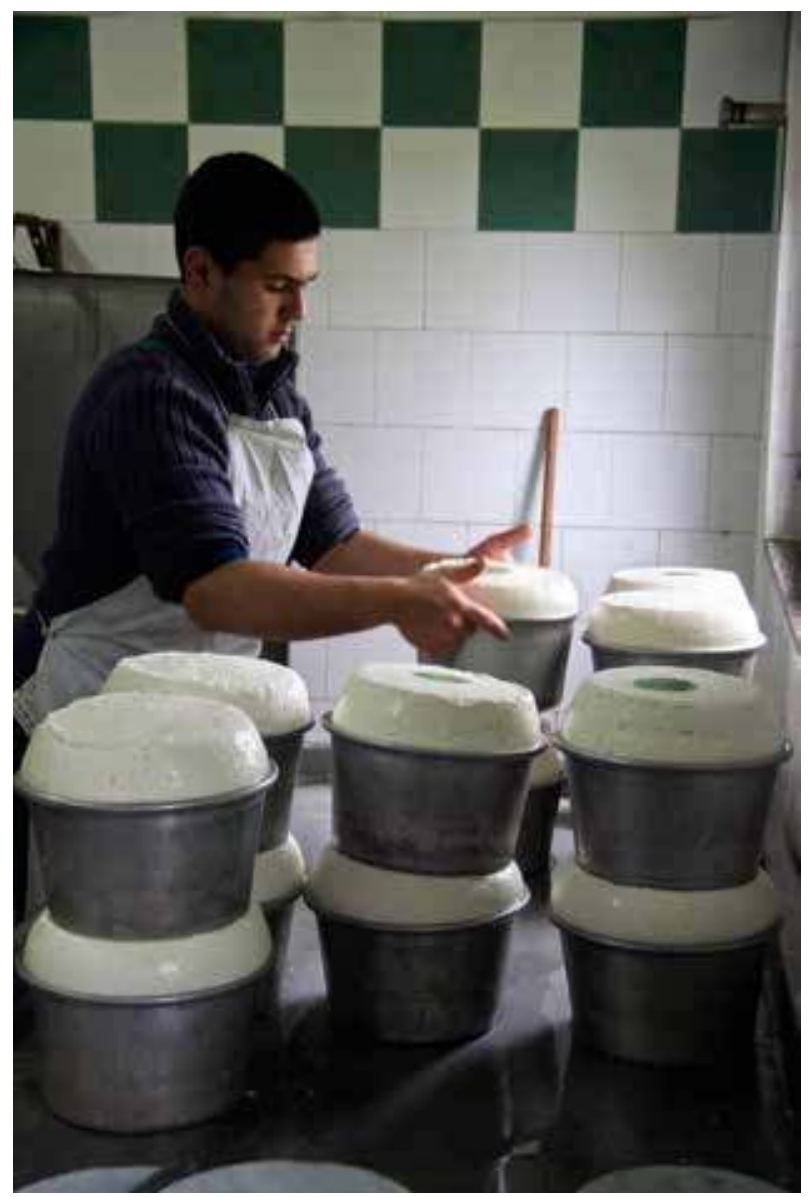

5. La marque de caséine, qui certifie l'appartenance à la AOC Fiore Sardo, avec le code du producteur, est apposée sur les formes.

La marque est délivrée par le «Consorzio di tutela » (Groupement de défense) de l'AOC. lait cru et la pratique du fumage - est l'un des enjeux de cette lutte, et la conservation y joue un rôle particulier. Les procédures de production, d'affinage et donc de conservation -en partie détaillées dans les cahiers des charges de l'AOC- requièrent en effet des lieux, des temps et des techniques bien précis. Elles jouent un rôle important dans le façonnement de ces fromages, dans la production de leur valeur - qui ne se limite pas à la sphère économique -, et dans leur mise en circulation (Graeber 2001, Harvey, McMeekin \& Warde 2004).

Pour opérer un premier niveau de distinction entre le Fiore Sardo produit par les bergers et le Fiore Sardo issu des fromageries industrielles, les bergers producteurs (en même temps membres du «Consorzio di tutela», Groupement de défense de l'AOC) avaient constitué en son sein un second groupement, doté d'un rôle juridique différent, le «Consorzio dei pastori storici produttori» (Groupement des bergers producteurs historiques). La finalité de ce second groupement était de rendre visible la spécificité de leur production de Fiore Sardo par rapport à celle des industriels, en vendant le produit sous une étiquette commune qui indiquait le nom du producteur à côté de celui du «Consorzio dei pastori storici produttori» et de celui de l'AOC. Ce dispositif, qui n’avait pas de réelle existence juridique, a perdu de son efficacité au fil des années. Entre-temps, un autre dispositif avait vu le jour: la sentinelle Slow Food. Lanalyse de la place qu'occupe ce fromage dans les actions de Slow Food, notamment à travers le label de sentinelle, et celle des relations que Slow Food entretient avec les producteurs bergers permettent d'illustrer la complexité des enjeux qui s'agrègent autour de la régulation de ce fromage, et qui sont directement liés à la définition des techniques de production et de conservation ${ }^{7}$. 


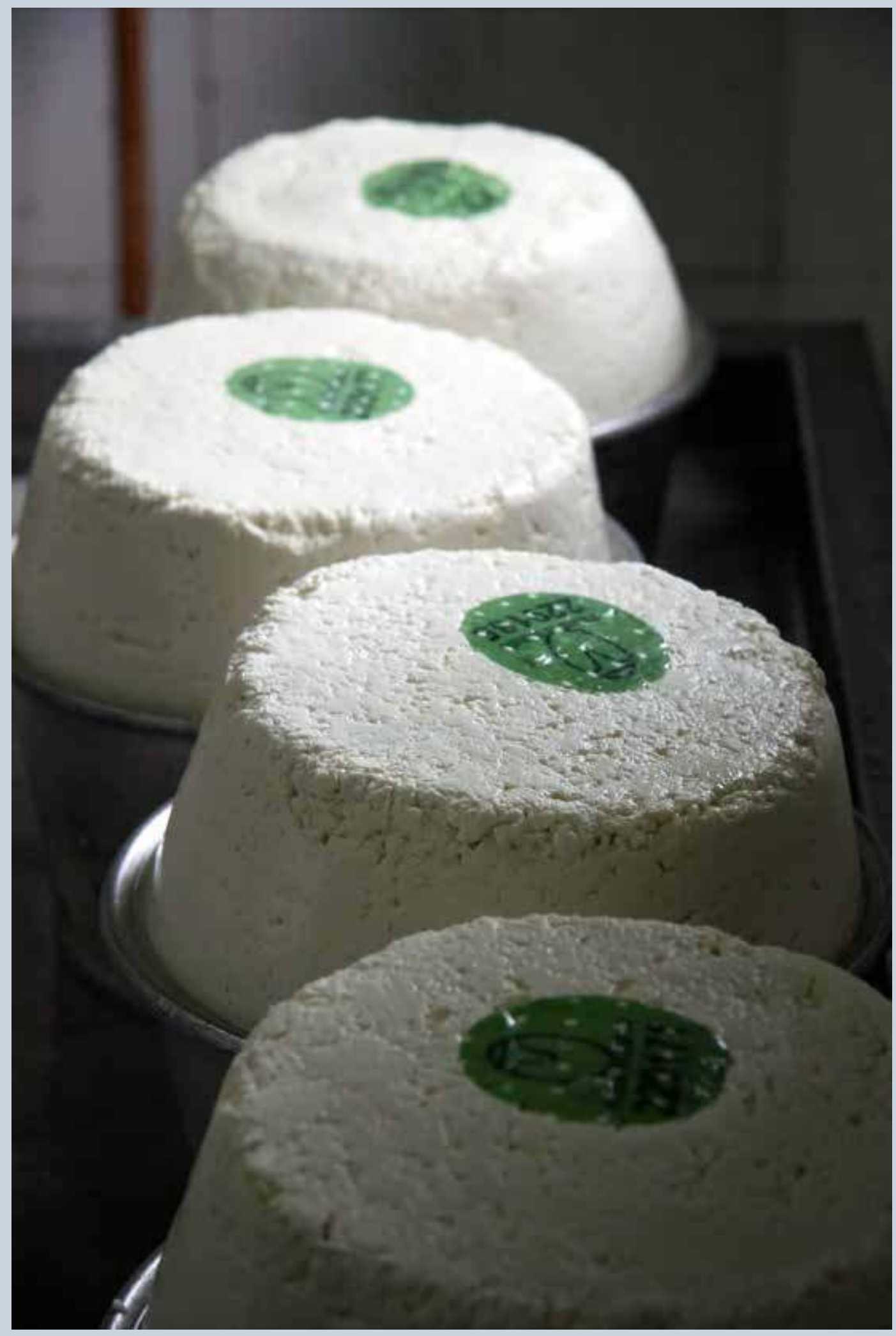

6. Une fois le petit-lait éliminé et les meules salées, celles-ci sont prêtes pour le fumage puis pour être entreposées dans la cave où elles resteront au moins trois mois. 
7. La phase du fumage intervient juste après la salaison, selon la procédure indiquée dans les cahiers des charges. Cette phase est considérée par les producteurs bergers comme l'un des éléments de distinction du fromage artisanal, et plus généralement du véritable Fiore Sardo.

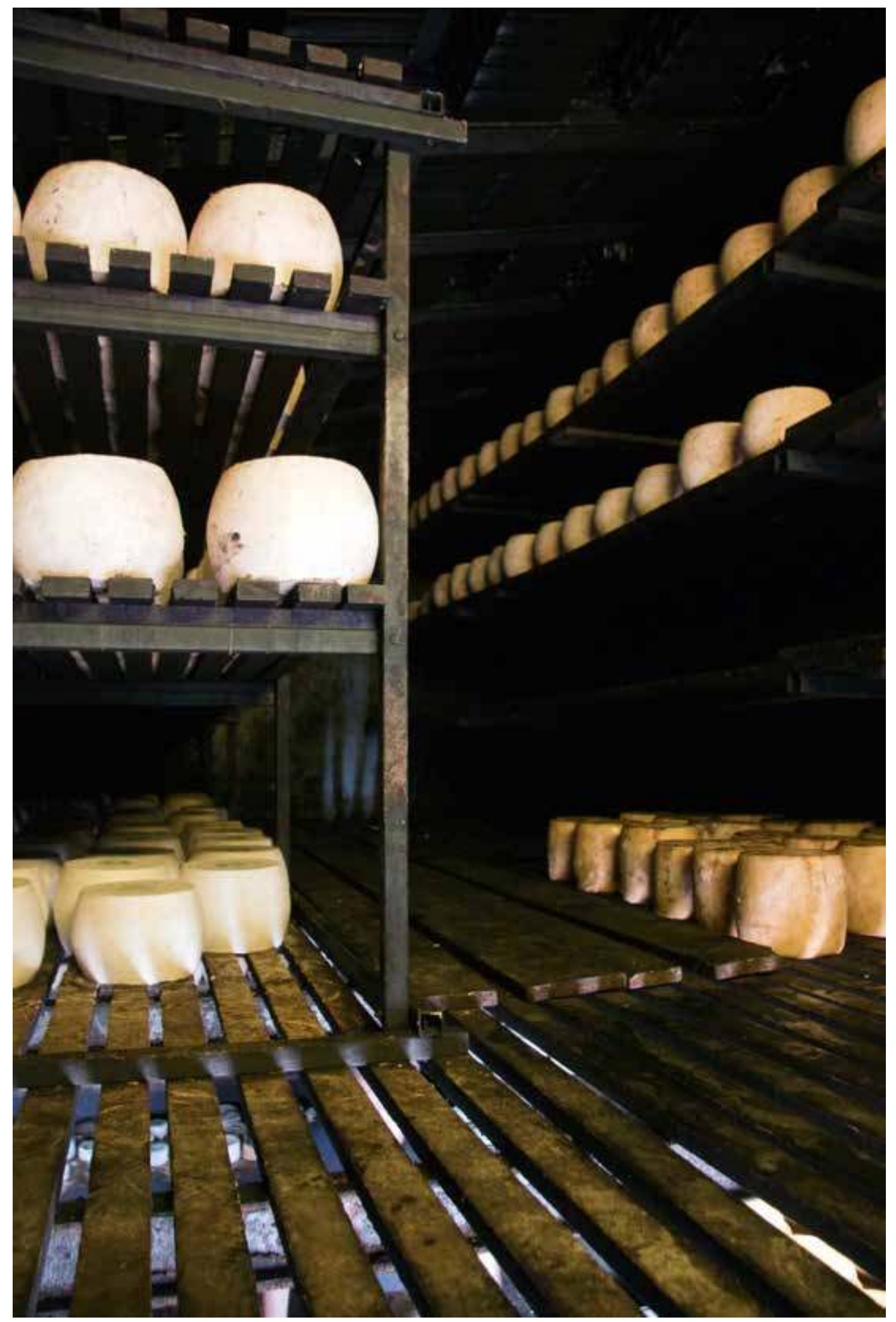




\section{Garnisons du goût et de la biodiversité agroalimentaire}

Au départ surtout intéressés par la défense de la qualité alimentaire et par les consommateurs, les leaders de Slow Food ont, au cours de ces vingt dernières années, élargi leurs actions à l'environnement et aux producteurs. La dimension transnationale de Slow Food ainsi que la capacité de ses membres et de ses responsables à intervenir - que ce soit par des projets concrets ou à travers des batailles médiatiques-sur l'ensemble de la filière alimentaire en font aujourd'hui un acteur reconnu et légitime des débats qui portent sur les problématiques de l'alimentation, de l'agriculture et de la pêche durables. Le «bon», le «propre» et le «juste» sont les trois piliers qui structurent les actions et la philosophie de Slow Food. Le «bon» fait référence aux caractéristiques organoleptiques des produits alimentaires. Le « propre» renvoie non seulement aux relations entre les processus de production et le maintien de l'environnement, mais aussi au respect de ce dernier par l'élimination des pesticides et des produits chimiques. Enfin, la notion de «juste» permet d'inclure les producteurs, leurs conditions de vie et leur survie économique parmi les intérêts prioritaires des membres du mouvement ${ }^{8}$. La production fromagère est l'un des centres d'intérêt les plus anciens de Slow Food. L'une des batailles historiques du mouvement est celle menée pour la défense des fromages au lait cru.

Le fromage au lait cru semble exprimer les valeurs historiques, paysagères et organoleptiques que le mouvement Slow Food s'attache à défendre non seulement à travers des actions de valorisation, mais aussi par des batailles politiques menées à différentes échelles. Dans le manifeste que leur a dédié le mouvement, les fromages au lait cru deviennent l'étendard d'une nouvelle manière de concevoir la production alimentaire, porteuse de connaissances, de valeurs et de différences. Pour Slow Food, la pasteurisation et l'utilisation des ferments sont synonymes d'uniformisation et de perte de la «biodiversité agroalimentaire», notion que le mouvement a introduite à partir des années 1990. Derrière ces dichotomies (lait cru contre pasteurisation, spécificités et diversité contre uniformisation) se dessinent les contours du champ de la régulation de la production dans lequel le mouvement essaie de se positionner comme acteur légitime, au même titre que des institutions comme le ministère de l'Agriculture italien.

Les «sentinelles Slow Food» sont un autre outil des batailles politiques du mouvement et un instrument de régulation: elles sont tout à la fois des produits et des projets de labellisation/ valorisation de petites productions considérées comme étant de «haute qualité » alimentaire et menacées de disparition, et elles visent à préserver simultanément des lieux de production et des activités économiques. Les sentinelles sont des prototypes, des modèles à suivre, non seulement pour les producteurs qui s'engagent à respecter un cahier des charges, mais aussi pour les autres producteurs (Siniscalchi 2010). Rédigé conjointement par les responsables de Slow Food et par les producteurs qui souhaitent bénéficier du label, le cahier des charges précise les modalités de production (lieux, temps, techniques de production, saisonnalité, race ou espèce, etc.) et définit ainsi, dans la pratique, les critères du bon, du propre et du juste pour la production concernée 9 .

La sentinelle Slow Food du Fiore Sardo est née au début des années 2000, de la volonté des bergers producteurs d'instaurer un degré supplémentaire de qualité et de distinction à l'intérieur de l'AOC. Si les contraintes imposées par le cahier des charges de la sentinelle, bien plus strictes que 
celles de l'AOC, ont fait qu'une partie de ces producteurs n’a pas pu, ou n’a pas voulu, adhérer à la sentinelle, tous les producteurs bergers bénéficient aujourd'hui indirectement de son existence.

La dimension collective, la valorisation des savoirs et de leur transmission (cf. Hermitte 2001) rapprochent ces deux dispositifs. En revanche, si les AOC protègent juridiquement une dénomination et définissent précisément une aire de production, le label des sentinelles n’assure pas une protection juridique aux produits ni à leur dénomination. Ce label est une marque privée et un outil de valorisation non pérenne, destiné à attirer l'attention des consommateurs, des producteurs et des élus sur un produit et sur les modalités spécifiques de sa production. Quand une sentinelle est créée à l'intérieur d'une $\mathrm{AOC}$, c'est-à-dire dans un espace déjà labellisé, l'AOC en général ou certains de ses aspects font l'objet de contestation, et la sentinelle devient l'outil de cette contestation. C'est dans cette dernière configuration que se situe la sentinelle du «Fiore Sardo dei Pastori ». Le cahier des charges de la sentinelle limite le label aux producteurs bergers qui travaillent avec le lait cru de leurs troupeaux. Il précise notamment que l'affinage doit durer minimum quatre mois, et que tout produit anti-moisissure et synthétique est interdit pendant toute la période de l'affinage. La volonté affichée par les responsables de Slow Food et par les producteurs est de singulariser (cf. Karpik 2007) le «Fiore» des bergers par rapport à celui des autres producteurs et notamment les industriels.
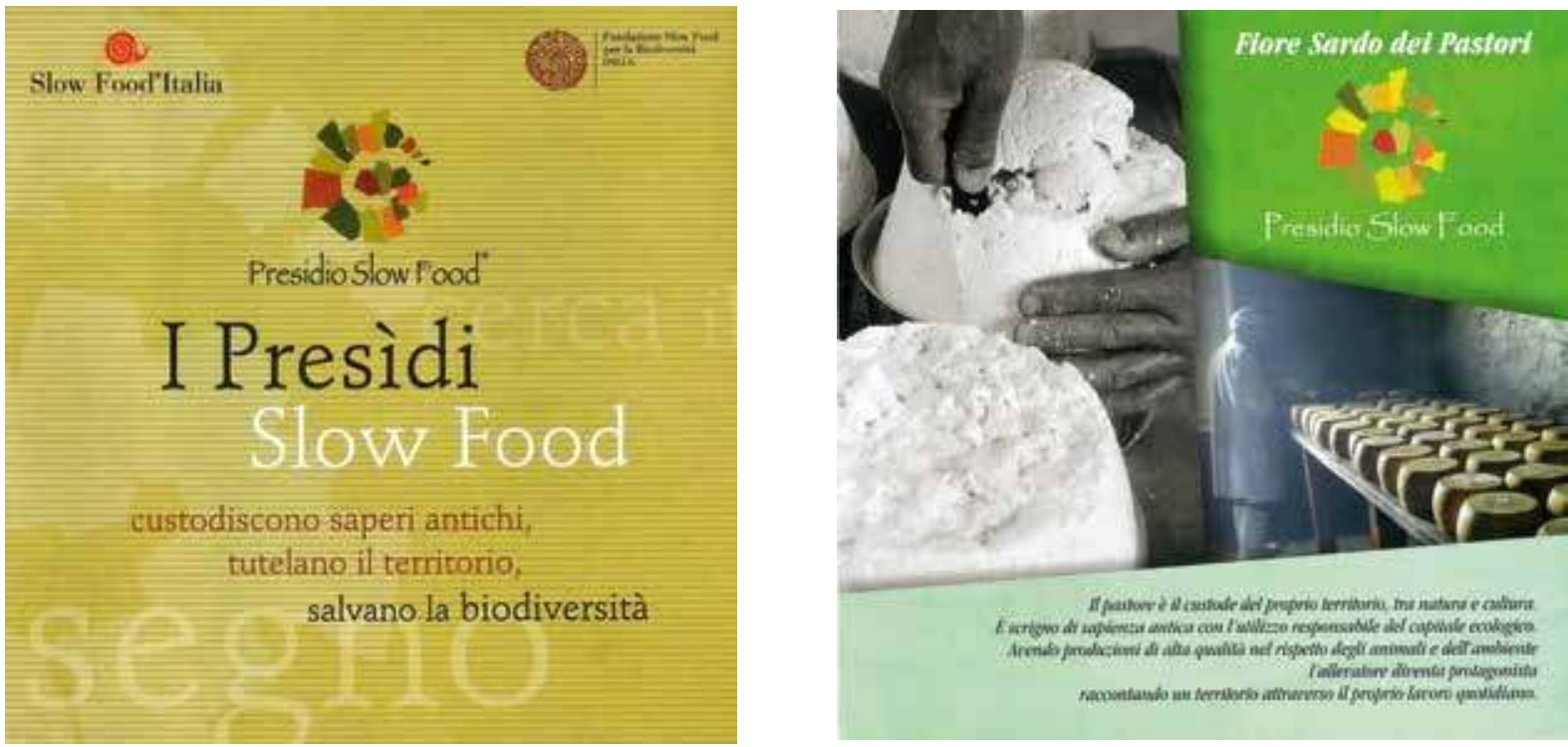

8. Brochure des sentinelles Slow Food, avec le label en forme de spirale qui est apposé sur les produits: «les sentinelles Slow Food gardent les anciens savoirs, protègent le territoire, sauvent la biodiversité ». Dans l'abondant lexique élaboré par Slow Food, le terme utilisé dans le monde entier pour désigner les produits labellisés par le mouvement est celui de «presidio/presidia» (garnison). En France, le terme choisi a été celui de «sentinelles ». J'aborde dans un autre texte (Siniscalchi 2017) les questions relatives à la traduction de la philosophie du mouvement.

9. «Le berger est le gardien de son propre territoire, entre nature et culture. Il est le coffre-fort d'une sagesse ancienne et il utilise de manière responsable le capital écologique. Avec des productions de haute qualité, qui respectent les animaux et l'environnement, l'éleveur devient le protagoniste qui raconte le territoire à travers son travail quotidien», affirme la brochure d'un producteur de Fiore Sardo dei Pastori (Fiore Sardo des bergers). 


\section{Les enjeux et les contradictions de la conservation}

Du point de vue organoleptique, pour les bergers producteurs, la conservation permet de modifier dans le temps la saveur de ce fromage et de le façonner ainsi en tant que véritable Fiore Sardo: d'abord par le fumage, puis par l'affinage à l'intérieur des caves où, pendant des mois, les meules sont régulièrement graissées et retournées. Pour pouvoir bénéficier de l'appellation d'origine, les fromages doivent être affinés et conservés pendant une période d'au moins 3 mois; de fait, le goût du fromage arrive à juste maturation entre 6 et 10 mois.

Puisqu'il s'agit d'un fromage au lait cru, la conservation et ses techniques permettent ensuite de le stocker dans la durée afin de pouvoir le vendre (idéalement) au bon moment, c'est-à-dire quand son prix de marché est le plus élevé. Pour les producteurs bergers, cette période de conservation - qui est faite de temps, de techniques, de conditions climatiques, de lieux de stockage - permet donc de construire la valeur de marché, mais aussi la valeur politique du Fiore Sardo. Si la notion de valeur, comme le souligne Graeber (2001), est utilisée pour indiquer à la fois les processus de valorisation, le prix de marché et la valeur en tant que «différence significative» par rapport à quelque chose d'autre, dans le cas du Fiore Sardo ces trois significations sont coprésentes et contribuent à définir sa valeur (croissante ou décroissante) sur le marché ainsi que sa valeur politique.

Du point de vue des commerçants et des distributeurs, la possibilité de conserver ce fromage permet sa mise en vente hors du territoire de la Sardaigne. Les Pouilles sont sa première destination commerciale depuis des décennies: les intermédiaires et les commerçants des Pouilles, en effet, ont appris dans le temps à négocier avec les producteurs de Sardaigne. Leur capacité à acheter en une seule fois de grandes quantités de fromage - par rapport à d'autres lieux commerciaux qui acquièrent par petites quantités afin de ne pas devoir gérer les stocks et les invendus - a contribué à construire leur pouvoir sur le marché du Fiore Sardo :

«La Bourse du Fiore Sardo se trouve à Lecce [dans les Pouilles]. C'est Lecce qui fait le prix chaque année. Si le prix est élevé à Lecce, il est élevé partout. Si le prix baisse à Lecce, il baisse partout parce que $70 \%$ du Fiore Sardo va dans les Pouilles [...] S'il reste encore du [fromage] vieux, ils achètent le nouveau à des prix dérisoires [...]. Et quand tu as un stock [de fromage qui peut être mis sur le marché] tu dois le vendre.» (Stefano S., producteur berger, 26/04/2011).

Plus qu'à des banques - métaphore souvent utilisée en raison du prix de certains fromages qui se prêtent à des affinages longs -, les caves du Fiore Sardo des bergers producteurs peuvent être comparées à des coffres-forts à durée déterminée. En effet, la durée de conservation semble accroître le prix de certains fromages sur le marché: c'est par exemple le cas du Parmigiano Reggiano ou encore du Bitto, dont l'affinage peut durer jusqu'à dix ans. En réalité, et plus particulièrement pour le Fiore Sardo, l'affinage repose sur un équilibre fragile entre temps et goût: il s'agit d'un processus pendant lequel le fromage continue de se transformer du point de vue de la fermeté de la pâte, qui devient plus dure et plus foncée, et du goût, qui devient plus piquant et plus salé. 


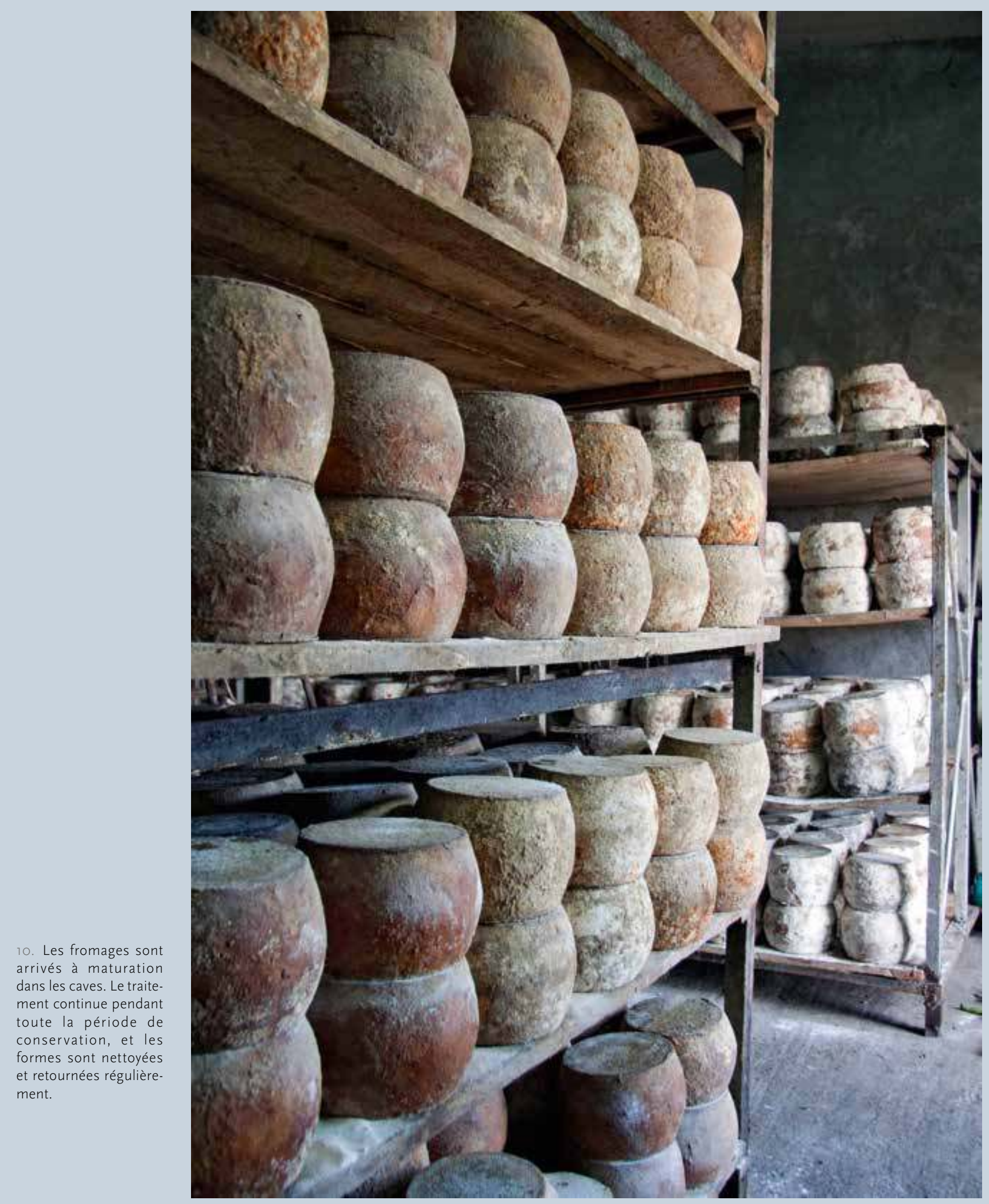


Si la période d'affinage se prolonge trop - en cas de non-vente - le goût du Fiore Sardo devient trop fort. Bien que sur certains marchés il soit vendu et utilisé comme fromage à râper, précisément pour ce goût intense et marqué, son prix commence alors à décroître. Seuls certains producteurs parviennent à utiliser à leur propre avantage le vieillissement du fromage, en cumulant plusieurs signes de qualité (AOC, label biologique, sentinelle Slow Food), l'accès à des marchés spécifiques et des conditions matérielles particulières (un grand troupeau et des grandes caves), autant de conditions qui ne sont pas partagées par la plupart des producteurs. C'est à ce moment précis que les grossistes peuvent faire jouer leur pouvoir d'achat pour proposer des prix plus bas, non seulement sur les fromages de l'année en cours, mais surtout sur les stocks de l'année précédente, en les acquérant en bloc. En effet, la gestion des stocks et des caves est coûteuse. Les meules non vendues constituent un capital bloqué, aussi bien pour les gros producteurs que pour les petits, et demandent un surplus de travail, car le fromage continue de se transformer et son traitement doit continuer pendant toute la période de conservation des meules. Bien que le problème de la gestion des stocks soit le même pour les producteurs industriels et pour les producteurs bergers, pour ces derniers, le fait d'avoir encore des produits non vendus quand la production de l'année suivante est à son maximum peut provoquer des difficultés économiques difficiles à surmonter. En effet, outre les problèmes d'espace de stockage, les producteurs bergers produisent souvent une seule variété de fromage et ne peuvent pas différencier leurs ventes et donc leurs entrées, comme le font les entreprises industrielles. Ils ne disposent pas non plus des systèmes de réfrigération dont se sont dotés les industriels: soumises aux aléas du climat, les meules de fromage peuvent voir leurs éventuels défauts s'accentuer. Dans ce cas, les intérêts des bergers producteurs et ceux de Slow Food se rencontrent à nouveau.

\section{Résistance fromagère}

En 2010, pour aider les producteurs de la sentinelle Fiore Sardo à faire face à une année particulièrement dure où les prix étaient au plus bas, Slow Food avait lancé la campagne «Resistenza casearia» (Résistance fromagère). Pendant plusieurs semaines, au siège de Bra, les meules de Fiore Sardo invendues et arrivées de Sardaigne, empilées dans un local, étaient emballées par l'un des employés, normalement chargé des relations avec les producteurs des sentinelles et devenu pour l'occasion magasinier, et elles étaient envoyées dans les différentes régions d'Italie où les adhérents de Slow Food en avaient fait la commande. Pour ces derniers, il s'agissait de s'impliquer activement dans l'une des actions du mouvement tout en achetant un produit de qualité qui restait à un prix inférieur à celui du marché. Pour les producteurs, l'opération permettait d'écouler, à temps et à un prix trois fois plus élevé que celui proposé par les commerçants, le stock de fromage que quelques mois de plus passés dans les caves auraient rendu invendable. 
Cette opération de vente directe n'a en apparence rien rapporté à Slow Food, puisqu'aucun pourcentage n'a été retenu sur le prix de vente, à part celui des frais d'emballage et d'expédition. En réalité, les gains ont été de nature politique: le Fiore Sardo est devenu un nouvel outil des batailles du mouvement. Cette campagne et l'écho médiatique qui l'a accompagnée et soutenue ont contribué à conforter le rôle politique de Slow Food dans le champ de la production et de la régulation alimentaire, ainsi que son image de soutien des petits producteurs.

Le Fiore Sardo est non seulement un produit «vivant» qui continue de se transformer dans le temps, mais aussi une marchandise soumise aux aléas du marché qui font apparaître plus clairement les enjeux et les contradictions de la conservation du fromage. Le goût du Fiore Sardo, qui résulte de procédures spécifiques de production allant du mode d'élevage (les brebis pâturent en montagne) aux techniques de production (lait cru) et de conservation (fumage et affinage), devient un «goût politique» qui s'oppose au goût «standardisé » des produits industriels. Des organismes comme l'ONAF (Organizzazione Nazionale Assaggiatori di Formaggi - Organisme national de dégustateurs de fromages -) écrivent du Fiore Sardo que «le goût n'est pas toujours équilibré car il présente fréquemment - surtout quand l'affinage est plus avancé - un excès de notes "dures" (sapidité, acidité et amertume) qui, avec la forte granulosité de la pâte, ne le rendent pas très agréable comme "fromage de table" (fiche technique du Fiore Sardo, http://www.onaf.it/uploads/public/2401_fiore-sardo.pdf). En revanche, pour les bergers producteurs, le goût de leur fromage est l'un des indicateurs des modes spécifiques de production et de conservation et une manière de distinguer le véritable Fiore Sardo dans le champ conflictuel des productions dites typiques. Pour les adhérents de Slow Food, apprendre à apprécier ce fromage à travers les ateliers, les campagnes de promotion, les dégustations et l'éducation au goût promus par le mouvement, est aussi une manière de s'impliquer politiquement dans le soutien des petites productions menacées par l'agro-industrie. Dans ce sens, la valeur du Fiore Sardo qu'ils contribuent à construire est aussi «la manière dont les acteurs se représentent l'importance de leurs propres actions en tant que membres d'un ensemble plus ample [...]. Deuxièmement, cette importance est souvent conçue en termes comparatifs » (Graeber 2005 : 451, ma traduction). Pour Slow Food, ces actions sont un moyen de contribuer à construire la valeur de ce fromage et de bâtir autour de lui une économie (morale) spécifique. En effet, la défense du bon, du propre et du juste assume, pour les leaders et les adhérents du mouvement tout comme pour les bergers producteurs, une connotation morale: le bon ne fait pas simplement référence aux qualités organoleptiques mais aussi au respect de techniques et de savoirs, au rapport entre ces savoirs et un territoire, enfin à une rémunération juste pour les producteurs. Et la conservation, toujours, participe de ces processus. 


\section{Notes}

1. Créé il y a trente ans en Italie (dans la région du Piémont) comme association de défense du plaisir de la table et de la convivialité, contre la standardisation de la nourriture, Slow Food est aujourd'hui à la fois un mouvement et une association internationale de près de 100000 membres, implantés dans différents pays du monde. Tout en gardant la nourriture comme sujet central, les intérêts de ses responsables et de ses adhérents ainsi que les domaines d'intervention se sont élargis.

2. Mon approche ethnographique s'est déclinée en périodes longues de permanence sur le terrain, d'abord en France, puis en Italie dans le quartier général de Slow Food et auprès de ses instances internationales (environ trois ans d'enquête). Une phase de ces recherches a été consacrée aux producteurs liés à Slow Food, et parmi eux, aux producteurs de Fiore Sardo.

3. Les travaux sur ces questions incluent les études de culture matérielle intéressées aux techniques, aux savoirs et à leur transmission, tout comme les travaux attentifs aux performances touristiques ou encore les écrits - surtout français, italiens et nord-américains - sur les produits de terroirs. La limite d'une partie de cette littérature était le parti pris du consensus que les produits de terroir et les appellations d'origine semblaient devoir et pouvoir créer, les conflits étant à peine esquissés (cf. entre autres Bérard et Marchenay 1995). D'autres travaux continuent aujourd'hui à réfléchir aux dynamiques de mise en place des appellations et des indications géographiques ( $c f$. en France les travaux du CIRAD ou de l'INRA), mais à l'intérieur de cadres théoriques différents de celui adopté ici.

4. J'utilise le terme «vie» dans une acception plus ouverte que celle à laquelle renvoie le terme «biographie» tel que Kopitoff (1986) l'a défini. La notion de vie me permet d'inclure des acteurs et des échelles d'analyse différents ainsi que des dynamiques plus larges.

5. Ceci à partir de 1955. Appellation définie par la convention internationale de Stresa de 1951, et intégrée dans la loi italienne 125 du 10 avril 1954.

6. Élaboration de l'auteure à partir des données AGRIS («Azienda regionale per la ricerca in agricoltura, Regione autonoma di Sardegna ») relatives à la production de 2014, de 2015 et de 2016.

7. Ces dispositifs évoluent constamment et sont au cœur de tensions, voire de conflits, forts: j'aborde ces questions dans un texte, en cours de rédaction, dans le cadre du projet VIPOMAR. Cf. aussi Papa (2002).

8. J'ai déjà eu l'occasion d'analyser différents aspects de ce mouvement (cf. entre autres Siniscalchi 2013a, 2013b, 2014a, 2014b). Slow Food est une «machine» complexe, dotée de composantes hétérogènes. C'est dans une double perspective, politique et économique, que j'ai abordé l'étude de ce mouvement.

9. Derrière ces accords se cachent parfois des incompréhensions et des frictions. Je ne rentrerai pas ici dans les détails de ces dynamiques, qui rappellent que les produits de terroir sont loin d'être consensuels, comme la littérature spécialisée semble parfois le laisser entendre.

\section{Remerciements}

Une partie des éléments présentés dans ce texte est issue d'une recherche en cours sur la vie politique des fromages de brebis en Sardaigne, dans le cadre de l'ANR «VIPOMAR. La vie politique des marchandises» (Projet ANR 14-CE29-0006-01). Une autre partie est issue des recherches que Valeria Siniscalchi a menées sur le mouvement Slow Food à partir de 2006. Un remerciement aux producteurs de Fiore Sardo, aux responsables et aux membres de Slow Food, et à tous les amis qui ont accueilli avec confiance cette recherche en Sardaigne. Un remerciement particulier à Geneviève Marotel pour les échanges riches et pour la relecture attentive de ce texte et aux relecteurs anonymes pour leurs retours sur une première version de cet article. 


\section{Les auteurs}

Maître de conférences (HDR) à l'EHESS (Centre Norbert Elias), Valeria Siniscalchi a mené ses premières recherches en Italie du Sud entre 1990 et 2000 (Frammenti di economie. Ricerche di antropologia economica in Italia, Pellegrini 2002). Après un terrain dans les Hautes-Alpes sur les rapports entre économie, pouvoir et identités dans les espaces protégés, depuis une dizaine d'années, elle travaille sur les mobilisations dans le domaine de la production et de la consommation alimentaire (Food Activism: Agency, Democracy and Economy, avec C. Counihan 2014). Elle a mené une enquête ethnographique sur le mouvement international Slow Food (Slow Food: The Economy and Politics of a Global Movement, en préparation chez Bloomsbury). Dans ses recherches actuelles sur la régulation et la circulation des productions fromagères, elle s'intéresse aux processus de transformation de l'économie et à leur dimension politique.

Entre 1975 et 1993, Franco Zecchin réalise un long travail photographique, socialement engagé sur la mafia, la corruption politique et les conditions sociales en Sicile. En 1988, il devient membre «nominé » de l’Agence Magnum. Entre 1989 et 1991, il conduit une enquête photographique sur les rapports entre pollution industrielle et santé publique en Silésie (Pologne). Dans les années 1990, il travaille sur le nomadisme et l'utilisation des ressources environnementales. Aujourd'hui, il continue à explorer les rapports entre appropriation du territoire et pratiques sociales à travers la photographie. Parmi ses publications, Nomades (avec la collaboration de P. Bonte et H. Guillaume, Éditions de la Marinière 1998), Chroniques Siciliennes (avec L. Battaglia, Centre National de la Photographie 1989, Actes Sud 2000), Dovere di Cronaca (avec L. Battaglia, Peliti Associati 2006), Abitare il silenzio. Un'antropologa in clausura (texte de F. Sbardella, Viella 2015). Ses photos figurent dans la collection de l'International Museum of Photography de Rochester, du MOMA de New York et de la Maison Européenne de la Photographie à Paris.

\section{Iconographie}

Image d'ouverture. Un troupeau de brebis de race sarde. $55 \%$ des brebis italiennes sont élevées en Sardaigne pour un total d'environ 3200000 brebis (de race sarde) présentes dans l'île. (C) Franco Zecchin.

\section{Références}

Appadurai, A. 1986 «Introduction: commodities and the politics of value $»$ in A. Appadurai dir. The Social Life of Things: Commodities in Cultural Perspective. Cambridge: Cambridge University Press: 3-63.

Bérard, L. \& P. Marchenay 1995 «Lieux, temps et preuves. La construction sociale des produits de terroir», Terrain 24: 153-164.

Graeber, D. 2001 Toward an Anthropological Theory of Value: The False Coin of Our Own Dreams. New York: Palgrave-Macmillan.

- 2005 «Value: Anthropological theories of value» in J. Carrier dir. A Handbook of Economic Anthropology. Cheltenham: Edward Elgar: 439-454.

Harvey, M., McMeekin, A. \& A. Warde dir. 2004 Qualities of Food. Manchester/New York: Manchester University Press.

Hermitte, M.-A. 2001 «Les appellations d'origine dans la genèse des droits de propriété intellectuelle»,
8. (C). Busso / Slow Food.

9. (C) Slow Food.

Crédits pour l'ensemble des images: @ Franco Zecchin.

Études, recherches, systèmes agraires, développement 32: 195-207.

Karpik, L. 2007 Léconomie des singularités. Paris: Gallimard. Kopytoff, I. 1986 «Cultural biography of things. Commoditisation as process $»$ in A. Appadurai dir. The Social Life of Things: Commodities in Cultural Perspective. Cambridge: Cambridge University Press: 64-91.

McKeown R. 2016 «Fiore Sardo» in C. Donnelly dir. The Oxford Companion to Cheese. Oxford: Oxford University Press : 272-273.

Meneley, A. 2007 «Like an Extra Virgin», American Anthropologist 109 (4) : 678-687.

Papa, C. 2002 «Il prodotto tipico come ossimoro: il caso dell'olio extravergine d'oliva umbro » in V. Siniscalchi dir. Frammenti di economie. Ricerche di antropologia economica in Italia. Cosenza: Luigi Pellegrini Editore: 159-191. 
Paxson, H. 2013 The life of Cheese: Crafting Food and Value in America. Berkley/Los Angeles/London: University of California Press.

Siniscalchi, V. 2000 «Il dolce paese del torrone. Economia e storia in un paese del Sud», Meridiana 38-39: 199-222.

— 2010 «Regimi di singolarità e politiche della ripetizione», La Ricerca Folklorica 61 : 125-134.

- 2013a «Environment, regulation and the moral economy of food in the Slow Food movement », Journal of Political Ecology 20 : 295-305.

- 2013b «Pastori, attivisti e mercato. Pratiche economiche e logiche politiche nei Presidi Slow Food», Voci. Annuale di Scienze Umane X: 173-182.
- 2014a «Slow Food activism between politics and economy» in C. Counihan \& V. Siniscalchi dir. Food Activism: Agency, Democracy and Economy. London: Bloomsbury: 225-241.

— 2014b «La politique dans l'assiette: restaurants et restaurateurs dans le mouvement Slow Food en Italie», Ethnologie française XLIV, 1 : 73-83.

— 2017 «Slow Food: les politiques locales d'un mouvement international» in T. Grillot \& S. Gacon dir. Manger autrement. Paris: PUF : 63-85.

Weiss, B. 2016 Real Pigs: Shifting Values in the Field of Local Pork. Durham and London: Duke University Press.

Wilk, R. 2006 «From wild weeds to artisanal cheese» in R. Wilk dir. Fast Food/Slow Food: The Cultural Economy of the Global Food System. Lanham, MD: Altamira: 13-28.

\section{Pour citer l'article}

Siniscalchi, V. \& F. Zecchin 2018 «Conservation et production de la valeur du Fiore Sardo. Producteurs, commerçants et activistes dans la régulation de la typicité d'un fromage italien», TechniquesE्Eulture 69 «Le temps des aliments», p. $56-73$. 